\title{
On the directed Oberwolfach Problem with equal cycle lengths
}

\author{
Andrea Burgess \\ Department of Mathematics \\ Ryerson University \\ Toronto, ON, Canada \\ andrea.burgess@ryerson.ca
}

\author{
Mateja Šajna \\ Department of Mathematics and Statistics \\ University of Ottawa \\ Ottawa, ON, Canada \\ msajna@uottawa.ca
}

Submitted: Dec 15, 2012; Accepted: Jan 9, 2014; Published: Jan 24, 2014

Mathematics Subject Classifications: 05B30, 05C20; 05C38, 05C51, 05C70

\begin{abstract}
We examine the necessary and sufficient conditions for a complete symmetric digraph $K_{n}^{*}$ to admit a resolvable decomposition into directed cycles of length $m$. We give a complete solution for even $m$, and a partial solution for odd $m$.
\end{abstract}

Keywords: Directed Oberwolfach Problem; complete symmetric digraph; resolvable directed cycle decomposition; Mendelsohn design

\section{Introduction}

In this paper, we consider the problem of decomposing the complete symmetric digraph $K_{n}^{*}$ into spanning subgraphs, each a vertex-disjoint union of directed cycles of length $m$. In other words, we are interested in a resolvable decomposition of $K_{n}^{*}$ into directed $m$-cycles. In the design-theoretic literature, such decompositions have also been called Mendelsohn designs [9].

This problem can be considered as the directed version of the well-known Oberwolfach Problem with equal cycle lengths, which was completely solved in $[3,4]$. The popular form of the Oberwolfach Problem asks whether it is possible to have $n$ conference participants (where $n$ is odd) sit down for dinner at circular tables, each table having space for $m$ participants, so that each person gets to dine next to each other person exactly once over the course of several (that is, $\frac{n-1}{2}$ ) nights. The directed version would then be asking whether such an arrangement might be possible so that each participant gets to sit to the right of each other participant exactly once.

It is obvious that $K_{n}^{*}$ admits a resolvable decomposition into directed 2-cycles if and only if $n$ is even. In the case of cycles of length 3, the problem has been solved by 
Bermond, Germa, and Sotteau [7], and in the case of cycles of length 4, by Bennett and Zhang [5], except for one missing case $(n=12)$ filled in by Adams and Bryant [1].

Theorem 1. [7] There exists a resolvable decomposition of $K_{n}^{*}$ into directed cycles of length 3 if and only if 3 divides $n$ and $n \neq 6$.

Theorem 2. [5, 1] There exists a resolvable decomposition of $K_{n}^{*}$ into directed cycles of length 4 if and only if 4 divides $n$ and $n \neq 4$.

In this paper, we solve the problem completely for all even cycle lengths $m \geqslant 6$, and partially for odd cycle lengths $m \geqslant 5$, proving the following main result.

Theorem 3. Let $m$ and $n$ be integers with $5 \leqslant m \leqslant n$. Then the following hold.

1. Let $m$ be even, or $m$ and $n$ be both odd. Then there exists a resolvable decomposition of $K_{n}^{*}$ into directed cycles of length $m$ if and only if $m$ divides $n$ and $(m, n) \neq(6,6)$.

2. Let $m$ be odd and $n$ even. Then there exists a resolvable decomposition of $K_{n}^{*}$ into directed cycles of length $m$ whenever $n \equiv 0(\bmod 4 m)$, except possibly for $n=8 m$.

Furthermore, if there exists a resolvable decomposition of $K_{2 m}^{*}$ into directed cycles of length $m$, then there exists a resolvable decomposition of $K_{n}^{*}$ into directed cycles of length $m$ whenever $m$ divides $n$.

Proof. Clearly, $m \mid n$ is a necessary condition in all cases. The rest follows from Theorems 17 and 20.

This paper is organized as follows. In Section 2 we introduce the terminology and present some basic observations on resolvable cycle decompositions, while in Section 3 we state some previous results as well as prove a few technical lemmas that serve as the big tools in the proof of our main result. In Sections 4 and 5, respectively, we then present the bulk of the proofs for the two large cases, $m$ even and $m$ odd. Finally, in Section 6 we present some related results, namely, resolvable decompositions in which all but one of the resolution classes consist of vertex-disjoint directed $m$-cycles.

\section{Preliminaries}

In this paper, the term graph will mean a simple graph, and digraph will mean a directed graph with no loops or multiple arcs.

Definition 4. A collection $\left\{H_{1}, H_{2}, \ldots, H_{k}\right\}$ of subgraphs of a graph $G$ is called a decomposition of $G$ if the edge set of $G$ is a disjoint union of the edge sets of the graphs $H_{1}, H_{2}, \ldots, H_{k}$. If this is the case, we write $G=H_{1} \oplus H_{2} \oplus \ldots \oplus H_{k}$. Furthermore, if each of the graphs $H_{i}$ is isomorphic to a graph $H$, then $\left\{H_{1}, H_{2}, \ldots, H_{k}\right\}$ is called an $H$-decomposition of the graph $G$. 
Definition 5. Let $\mathcal{D}=\left\{H_{1}, H_{2}, \ldots, H_{k}\right\}$ be an $H$-decomposition of the graph $G$. A resolution class of $\mathcal{D}$ is a subset $\left\{H_{i_{1}}, H_{i_{2}}, \ldots, H_{i_{t}}\right\}$ of $\mathcal{D}$ with the property that the vertex sets of the graphs $H_{i_{1}}, H_{i_{2}}, \ldots, H_{i_{t}}$ partition the vertex set of $G$.

An $H$-decomposition $\mathcal{D}=\left\{H_{1}, H_{2}, \ldots, H_{k}\right\}$ of a graph $G$ is called resolvable if it can be partitioned into resolution classes of $\mathcal{D}$.

Clearly, any decomposition of a graph $G$ into spanning subgraphs is necessarily resolvable. Note that the above definitions naturally extend to directed graphs.

As usual, the symbols $K_{n}, \bar{K}_{n}$, and $K_{m, n}$ will denote the complete graph with $n$ vertices, the edgeless graph with $n$ vertices, and the complete bipartite graph with bipartition sets of cardinalities $m$ and $n$, respectively. In addition, for $n$ even, the symbol $K_{n}-I$ will denote the complete graph $K_{n}$ with the edges of a 1-factor $I$ removed. For any graph $G$, the symbol $G^{*}$ will denote the (symmetric) digraph obtained from $G$ by replacing each edge $u v$ by the two $\operatorname{arcs}(u, v)$ and $(v, u)$. Hence $K_{n}^{*}$ and $K_{m, n}^{*}$, respectively, will denote the complete symmetric digraph with $n$ vertices, and the complete bipartite symmetric digraph with bipartition sets of cardinalities $m$ and $n$.

By $C_{m}$ and $\vec{C}_{m}$ we shall denote the cycle of length $m$, and the directed cycle of length $m$, respectively. We shall use abbreviations $\mathrm{R} C_{m}$-D and $\mathrm{R} \vec{C}_{m^{-}}$- D to mean resolvable $C_{m^{-}}$ and $\vec{C}_{m}$-decompositions, respectively.

Recall that the wreath product $G<H$ of graphs $G$ and $H$ is obtained from $G$ by replacing every vertex $u$ of $G$ with a copy $H_{u}$ of the graph $H$, and for each edge $u v$ of $G$, inserting an edge between every vertex of $H_{u}$ and every vertex of $H_{v}$. Similarly, if $G$ and $H$ are digraphs, then the wreath product $G<H$ is a digraph obtained from $G$ by replacing every vertex $u$ of $G$ with a copy $H_{u}$ of the digraph $H$, and for each arc $(u, v)$ of $G$, inserting an arc from every vertex of $H_{u}$ to every vertex of $H_{v}$.

The following four observations are straightforward, hence the proofs are omitted. Note that the first three naturally extend to directed graphs.

Lemma 6. Let $G, H$, and $L$ be three graphs. Suppose $G$ admits a resolvable $H$-decomposition, and $H$ admits a resolvable $L$-decomposition. Then $G$ admits a resolvable L-decomposition.

Lemma 7. Let $\left\{H_{1}, H_{2}, \ldots, H_{k}\right\}$ be a decomposition of a graph $G$ into spanning subgraphs. Let $L$ be a subgraph of $G$ and suppose each graph $H_{i}$ admits a resolvable $L$-decomposition. Then $G$ admits a resolvable $L$-decomposition.

Lemma 8. Let $G$ and $H$ be graphs, and suppose there exists a resolvable $H$-decomposition of $G$. Then there exist a resolvable $H$-decomposition of $\bar{K}_{n} \prec G$, and a resolvable $H \curlyvee \bar{K}_{n}$ decomposition of $G \backslash \bar{K}_{n}$.

Lemma 9. Let $G$ be a graph, and $H$ a subgraph in $G$. If $G$ admits a resolvable $H$ decomposition, then $G^{*}$ admits a resolvable $H^{*}$-decomposition 


\section{Tools}

In some of our proofs in Sections $4-6$ we shall be referring to the following results.

Theorem 10. [4] There exists a resolvable $C_{m}$-decomposition of $K_{n}$ if and only if $n$ is odd and $m$ divides $n$.

Theorem 11. $[3,4,11]$ There exists a resolvable $C_{m}$-decomposition of $K_{n}-I$ if and only if $n$ is even and $m$ divides $n$.

Theorem 12. [2] There exists a $\vec{C}_{m}$-decomposition of $K_{n}^{*}$ if and only if $m$ divides $n(n-1)$ and $(m, n) \notin\{(4,4),(3,6),(6,6)\}$.

Theorem 13. [13] There exists a resolvable $C_{m}$-decomposition of $K_{n} \imath \bar{K}_{t}$ if and only if $m$ divides $n t, t(n-1)$ is even, $m$ is even if $n=2$, and $(m, n, t) \notin\{(3,3,2),(3,3,6),(3,6,2)$, $(6,2,6)\}$.

Our constructions in the case $m$ even will use the following lemmas as building blocks.

Lemma 14. Let $m \geqslant 4$ be an even integer, and $\beta$ a positive integer. Then there exists an $R \vec{C}_{m}-D$ of the graph $K_{\beta \frac{m}{2}, \beta \frac{m}{2}}^{*}$.

Proof. First, we prove the lemma for $\beta=1$. Let $k=\frac{m}{2}$, and let the bipartition of $V\left(K_{k, k}^{*}\right)$ be $\{X, Y\}$ with $X=\left\{x_{0}, x_{1}, \ldots, x_{k-1}\right\}$ and $Y=\left\{y_{0}, y_{1}, \ldots, y_{k-1}\right\}$. Then

$$
C^{0}=x_{0} y_{0} x_{1} y_{1} \ldots x_{k-1} y_{k-1} x_{0}
$$

is a directed cycle in $V\left(K_{k, k}^{*}\right)$. For $i=1,2, \ldots, k-1$, obtain a directed cycle $C^{i}$ from $C^{0}$ by adding $i$ (modulo $k$ ) to the subscripts of all vertices in part $Y$. It is not difficult to see that $\left\{C^{i}: i=0,1, \ldots, k-1\right\}$ is an $\mathrm{R} \vec{C}_{m}-\mathrm{D}$ of $K_{k, k}^{*}$.

Next, suppose $\beta \geqslant 2$. A 1 -factorization of $K_{\beta, \beta}$ yields a resolvable $K_{2}$-decomposition of $K_{\beta, \beta}$, and hence a resolvable $K_{2}^{*}$-decomposition of $K_{\beta, \beta}^{*}$ by Lemma 9. Consequently, there exists a resolvable $K_{2}^{*} \nmid \bar{K}_{\frac{m}{2}}$-decomposition of $K_{\beta, \beta}^{*} \imath \bar{K}_{\frac{m}{2}}$ by Lemma 8. Since $K_{2}^{*} \succ \bar{K}_{\frac{m}{2}}$ and $K_{\beta, \beta}^{*} \chi \bar{K}_{\frac{m}{2}}$ are isomorphic to $K_{\frac{m}{2}, \frac{m}{2}}^{*}$ and $K_{\beta \frac{m}{2}, \beta \frac{m}{2}}^{*}$, respectively, and the former digraph admits an $\mathrm{R} \vec{C}_{m}$-D by the first paragraph of this proof, Lemma 6 shows that so does the latter.

Lemma 15. Let $m \geqslant 4$ be an even integer, and $\alpha \geqslant 3, \beta \geqslant 1$ integers. Then there exists an $R \vec{C}_{m}-D$ of $C_{\alpha}^{*} \prec \bar{K}_{\beta m}$.

Proof. If $\alpha$ is even, a 1-factorization of $C_{\alpha}$ gives a resolvable $K_{2}$-decomposition of $C_{\alpha}$, and hence a resolvable $K_{2}^{*}$-decomposition of $C_{\alpha}^{*}$ by Lemma 9 . Lemma 8 then implies a

resolvable $K_{2}^{*} \imath \bar{K}_{\beta m}$-decomposition of $C_{\alpha}^{*} \imath \bar{K}_{\beta m}$. Since the former digraph is isomorphic to $K_{\beta m, \beta m}^{*}$, which admits an $\mathrm{R} \vec{C}_{m}$-D by Lemma 14 , the latter digraph also admits $\mathrm{R} \vec{C}_{m}-\mathrm{D}$ by Lemma 6. 


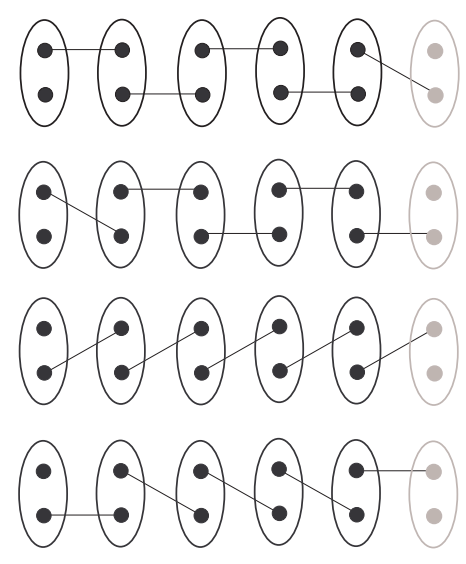

Figure 1: A resolvable $K_{2}$-decomposition of $C_{\alpha} \prec \bar{K}_{2}$ for $\alpha$ odd.

Next, assume $\alpha$ is odd. First, we show that $C_{\alpha}<\bar{K}_{2}$ admits a resolvable $K_{2}$-decomposition; that is, the edge set of $C_{\alpha}\left\langle\bar{K}_{2}\right.$ can be partitioned into pairwise disjoint perfect matchings. Let the vertex sets of $C_{\alpha}$ and $\bar{K}_{2}$ be $\left\{x_{0}, x_{1}, \ldots, x_{\alpha-1}\right\}$ and $\left\{y_{0}, y_{1}\right\}$, respectively. Then $C_{\alpha} \chi \bar{K}_{2}$ has vertices $\left(x_{i}, y_{j}\right)$, where $i=0,1, \ldots, \alpha-1$ and $j=0,1$. For convenience, we shall write $z_{i}^{j}$ for $\left(x_{i}, y_{j}\right)$. Note that $z_{i}^{j}$ is adjacent to $z_{s}^{t}$ if and only if $i-s \equiv \pm 1(\bmod \alpha)$. The following four perfect matchings of $C_{\alpha} \prec \bar{K}_{2}$ give the required decomposition (see Figure 1):

$$
\begin{aligned}
& M_{1}=\left\{z_{0}^{0} z_{1}^{0}, z_{1}^{1} z_{2}^{1}, z_{2}^{0} z_{3}^{0}, z_{3}^{1} z_{4}^{1}, \ldots, z_{\alpha-1}^{0} z_{0}^{1}\right\}, \\
& M_{2}=\left\{z_{0}^{0} z_{1}^{1}, z_{1}^{0} z_{2}^{0}, z_{2}^{1} z_{3}^{1}, z_{3}^{0} z_{4}^{0}, \ldots, z_{\alpha-1}^{1} z_{0}^{1}\right\}, \\
& M_{3}=\left\{z_{0}^{1} z_{1}^{0}, z_{1}^{1} z_{2}^{0}, z_{2}^{1} z_{3}^{0}, z_{3}^{1} z_{4}^{0}, \ldots, z_{\alpha-1}^{1} z_{0}^{0}\right\}, \\
& M_{4}=\left\{z_{0}^{1} z_{1}^{1}, z_{1}^{0} z_{2}^{1}, z_{2}^{0} z_{3}^{1}, z_{3}^{0} z_{4}^{1}, \ldots, z_{\alpha-1}^{0} z_{0}^{0}\right\} .
\end{aligned}
$$

Lemma 9 now implies that there exists a resolvable $K_{2}^{*}$-decomposition of $C_{\alpha}^{*} \imath \bar{K}_{2}$. Hence there exists a resolvable $K_{2}^{*} \succ \bar{K}_{\beta \frac{m}{2}}$-decomposition of $\left(C_{\alpha}^{*} \succ \bar{K}_{2}\right) \succ \bar{K}_{\beta \frac{m}{2}}$ by Lemma 8. Since $K_{2}^{*} \succ \bar{K}_{\beta \frac{m}{2}}$ is isomorphic to $K_{\beta \frac{m}{2}, \beta \frac{m}{2}}^{*}$, which admits an $\mathrm{R} \vec{C}_{m}$-D by Lemma 14 , and $\left(C_{\alpha}^{*} \prec\right.$ $\left.\bar{K}_{2}\right) \imath \bar{K}_{\beta \frac{m}{2}}$ is isomorphic to $C_{\alpha}^{*} \prec \bar{K}_{\beta m}^{2}$, we have the result.

The following lemma, except for $(m, \alpha)=(6,2)$, is a corollary of a special case of Liu's Theorem 13 [13]. Since the proof of the general result by Liu is long and involved, and our proof of this special case is short and transparent, we include it here.

Lemma 16. Let $m \geqslant 4$ be an even integer, and $\alpha \geqslant 2$ and $\beta \geqslant 1$ integers. Then there exists an $R \vec{C}_{m}-D$ of $K_{\alpha}^{*}<\bar{K}_{\beta m}$.

Proof. First assume $\alpha$ is even. Hence there exists a 1-factorization (that is, a resolvable $K_{2}$-decomposition) of $K_{\alpha}$. Consequently, there exist a resolvable $K_{2}^{*}$-decomposition of $K_{\alpha}^{*}$ (Lemma 9), and a resolvable $K_{2}^{*} \succ \bar{K}_{\beta m}$-decomposition of $K_{\alpha}^{*} \imath \bar{K}_{\beta m}$ (Lemma 8). Since $K_{2}^{*} \imath \bar{K}_{\beta m}$ is isomorphic to $K_{\beta m, \beta m}^{*}$, we have a resolvable $K_{\beta m, \beta m}^{*}$-decomposition of $K_{\alpha}^{*} \imath \bar{K}_{\beta m}$, and hence (by Lemmas 14 and 6 ) an $\mathrm{R} \vec{C}_{m}$-D of $K_{\alpha}^{*} \prec \bar{K}_{\beta m}$. 
Next suppose $\alpha$ is odd. Walecki's result [14], subsumed in Theorem 10, gives a (resolvable) $C_{\alpha}$-decomposition of $K_{\alpha}$, and Lemma 9 then implies a (resolvable) $C_{\alpha}^{*}$-decomposition of $K_{\alpha}^{*}$. Lemma 8 then gives us a resolvable $C_{\alpha}^{*} \imath \bar{K}_{\beta m}$-decomposition of $K_{\alpha}^{*} \prec \bar{K}_{\beta m}$. Since by Lemma 15 there exists an $\mathrm{R} \vec{C}_{m}$-D of $C_{\alpha}^{*} \prec \bar{K}_{\beta m}$, the result follows.

\section{Even cycle length}

The main purpose of this section is to prove the following theorem.

Theorem 17. Let $m \geqslant 6$ be an even integer and $\alpha$ a positive integer. Then $K_{\alpha m}^{*}$ admits an $R \vec{C}_{m}-D$ if and only if $(m, \alpha) \neq(6,1)$.

Proof. In the case of $\alpha=1$, Theorem 12 assures us of the existence of an $\mathrm{R}_{m^{-}}$D of $K_{\alpha m}^{*}$ for all $m \geqslant 8$, and of non-existence of an $\mathrm{R} \vec{C}_{6}-\mathrm{D}$ of $K_{6}^{*}$. Hence assume $\alpha \geqslant 2$.

First let $m \geqslant 8$. Decompose

$$
K_{\alpha m}^{*}=\left(\bar{K}_{\alpha} \prec K_{m}^{*}\right) \oplus\left(K_{\alpha}^{*} \prec \bar{K}_{m}\right) .
$$

Since $K_{m}^{*}$ admits an $\mathrm{R} \vec{C}_{m}$-D by Theorem 12 , so does $\bar{K}_{\alpha} \imath K_{m}^{*}$. And since $K_{\alpha}^{*} \imath \bar{K}_{m}$ admits an $\mathrm{R} \vec{C}_{m}$-D by Lemma 16 , we have an $\mathrm{R} \vec{C}_{m}$-D of $K_{\alpha m}^{*}$.

It remains to consider the case $m=6$. The challenge here is that, by Theorems 12 and 13 , respectively, there is no $\vec{C}_{6}$-decomposition of $K_{6}^{*}$ and no $\mathrm{R} C_{6}$-D of $K_{6,6}$.

Suppose $\alpha$ is even. If $\alpha=2$, we use Lemma 18 to find an $\mathrm{R}_{6}-\mathrm{D}$ of $K_{12}^{*}$. For $\alpha \geqslant 4$, first decompose

$$
K_{6 \alpha}^{*}=\left(\bar{K}_{\frac{\alpha}{2}} \succ K_{12}^{*}\right) \oplus\left(K_{\frac{\alpha}{2}}^{*} \succ \bar{K}_{12}\right) .
$$

Again using Lemma 18, we find an $\mathrm{R} \vec{C}_{6}$-D of $K_{12}^{*}$, and hence of $\bar{K}_{\frac{\alpha}{2}} \succ K_{12}^{*}$. To complete the construction, Lemma 16 gives us an $\mathrm{R} \vec{C}_{6}$-D of $K_{\frac{\alpha}{2}}^{*} \succ \bar{K}_{12}$.

Next, suppose $\alpha \geqslant 3$ is odd. Hence there exists a Hamilton cycle decomposition of $K_{\alpha}$. Consequently, we can decompose $K_{6 \alpha}^{*}$ into $C_{\alpha}^{*} \imath K_{6}^{*}$ and $\frac{\alpha-3}{2}$ copies of $C_{\alpha}^{*} \imath \bar{K}_{6}$. Now $C_{\alpha}^{*} \imath \bar{K}_{6}$ admits an $\mathrm{R} \vec{C}_{6}$-D by Lemma 15 , and an $\mathrm{R} \vec{C}_{6}-\mathrm{D}$ of $C_{\alpha}^{*} \succ K_{6}^{*}$ is constructed in Lemma 19.

Observe that the proof of Theorem 17 is independent of previous work (in particular, it makes no use of Theorem 13 by Liu) except the result on existence of a directed Hamilton cycle decomposition of $K_{m}^{*}$ (included in Theorem 12 and first proved by Tillson [15]).

Lemma 18. There exists an $R \vec{C}_{6}-D$ of $K_{12}^{*}$.

Proof. We label the vertices of $K_{12}^{*}$ by $x_{0}, x_{1}, \ldots, x_{5}, y_{0}, y_{1}, \ldots, y_{5}$. We shall call arcs of the form $\left(x_{i}, x_{i+d}\right)$ and $\left(y_{i}, y_{i+d}\right)$ arcs of pure left and pure right difference $d$, respectively, and arcs of the form $\left(x_{i}, y_{i+d}\right)$ and $\left(y_{i}, x_{i+d}\right)$ arcs of mixed left and mixed right difference $d$, respectively. All subscripts will be evaluated modulo 6. By the mirror image of a resolution class we shall mean the image under the permutation $\left(x_{0} y_{0}\right)\left(x_{1} y_{1}\right) \ldots\left(x_{5} y_{5}\right)$. 


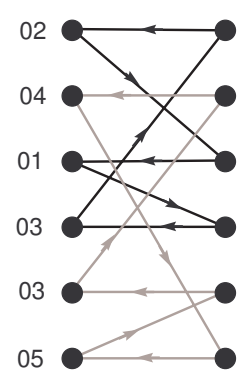

+ mirror image

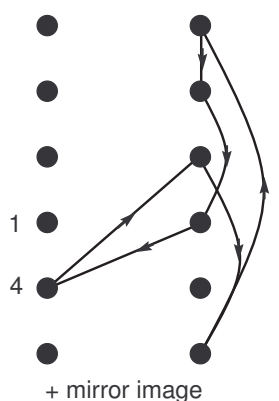

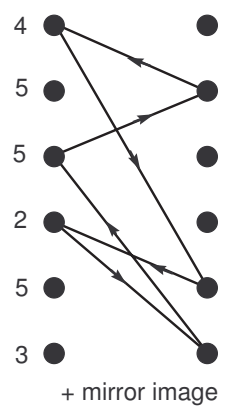

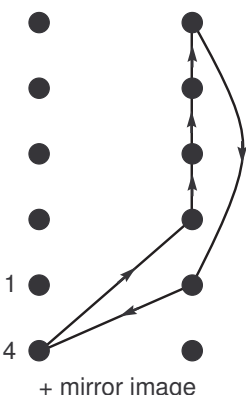

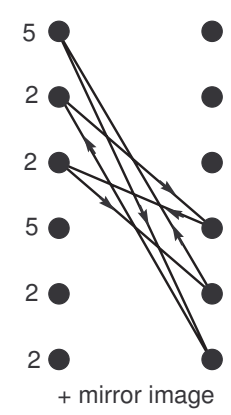
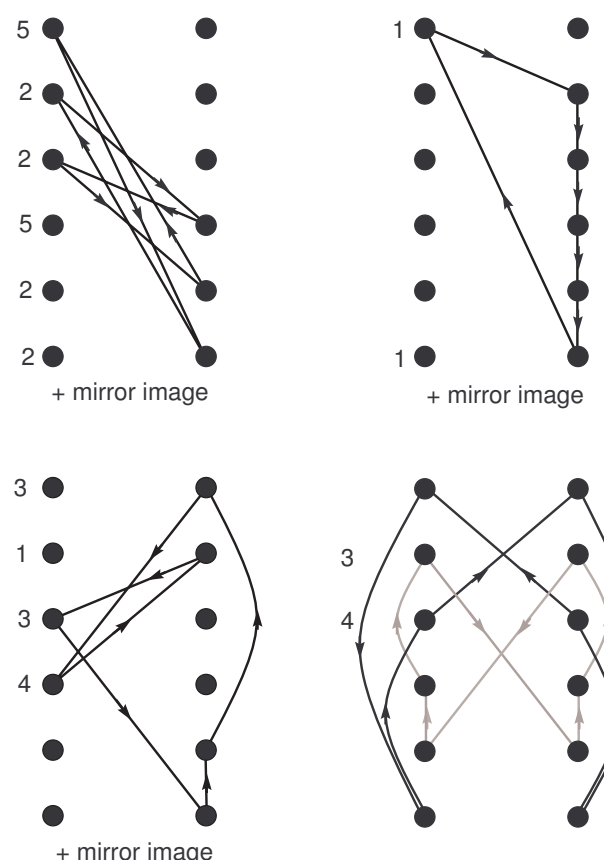

+ mirror image

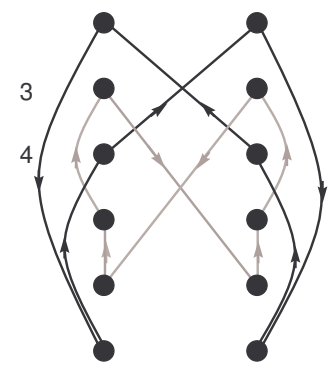

Figure 2: The first nine resolution classes in the $\mathrm{R} \vec{C}_{6}$-D of $K_{12}^{*}$.

The resolution classes in our decomposition are $R_{1}, \ldots, R_{11}$ defined below (see Figure 2).

$$
R_{1}=\left\{x_{0} y_{2} x_{2} y_{3} x_{3} y_{0} x_{0}, x_{1} y_{5} x_{5} y_{4} x_{4} y_{1} x_{1}\right\}
$$

and $R_{2}$ is its mirror image, that is,

$$
R_{2}=\left\{y_{0} x_{2} y_{2} x_{3} y_{3} x_{0} y_{0}, y_{1} x_{5} y_{5} x_{4} y_{4} x_{1} y_{1}\right\} .
$$

Observe that $R_{3}, \ldots, R_{8}$ all contain a directed cycle and its mirror image:

$$
\begin{aligned}
& R_{3}=\left\{x_{0} y_{4} x_{3} y_{5} x_{2} y_{1} x_{0}, y_{0} x_{4} y_{3} x_{5} y_{2} x_{1} y_{0}\right\}, \\
& R_{4}=\left\{x_{0} y_{5} x_{1} y_{3} x_{2} y_{4} x_{0}, y_{0} x_{5} y_{1} x_{3} y_{2} x_{4} y_{0}\right\}, \\
& R_{5}=\left\{x_{0} y_{1} y_{2} y_{3} y_{4} y_{5} x_{0}, y_{0} x_{1} x_{2} x_{3} x_{4} x_{5} y_{0}\right\}, \\
& R_{6}=\left\{y_{0} y_{1} y_{3} x_{4} y_{2} y_{5} y_{0}, x_{0} x_{1} x_{3} y_{4} x_{2} x_{5} x_{0}\right\}, \\
& R_{7}=\left\{y_{0} y_{4} x_{5} y_{3} y_{2} y_{1} y_{0}, x_{0} x_{4} y_{5} x_{3} x_{2} x_{1} x_{0}\right\}, \\
& R_{8}=\left\{y_{0} x_{3} y_{1} x_{2} y_{5} y_{4} y_{0}, x_{0} y_{3} x_{1} y_{2} x_{5} x_{4} x_{0}\right\},
\end{aligned}
$$

while $R_{9}$ is its own mirror image:

$$
R_{9}=\left\{x_{0} x_{5} x_{2} y_{0} y_{5} y_{2} x_{0}, x_{1} y_{4} y_{3} y_{1} x_{4} x_{3} x_{1}\right\} .
$$

The remaining two resolution classes are reversals of each other, and each consists of two directed cycles that are mirror images of each other and include only arcs of pure difference:

$$
R_{10}=\left\{x_{0} x_{2} x_{4} x_{1} x_{5} x_{3} x_{0}, y_{0} y_{2} y_{4} y_{1} y_{5} y_{3} y_{0}\right\}
$$


and

$$
R_{11}=\left\{x_{0} x_{3} x_{5} x_{1} x_{4} x_{2} x_{0}, y_{0} y_{3} y_{5} y_{1} y_{4} y_{2} y_{0}\right\} .
$$

Because of the symmetry, it is sufficient to verify that each arc of pure left difference and each arc of mixed left difference was used in exactly one directed 6-cycle. In Figure 2, the numbers on the left keep track of the arcs of mixed left difference used by the resolution classes $R_{1}, \ldots, R_{9}$. Observe that for each vertex $x_{i}$, each mixed left difference occurs exactly once. To account for all arcs of pure left (and hence right) difference, it is helpful to notice that in the union of the resolution classes $R_{1}, \ldots, R_{9}$, each arc $(u, v)$ occurs together with its opposite arc $(v, u)$. The rest of the verification is straightforward and left to the reader.

Lemma 19. For any odd integer $\alpha \geqslant 3$, there exists an $R \vec{C}_{6}-D$ of $C_{\alpha}^{*} \imath K_{6}^{*}$.

Proof. Let the vertex set of $C_{\alpha}^{*}\left\langle K_{6}^{*}\right.$ be $\left\{x_{j}^{(i)}: j \in \mathbb{Z}_{6}, i \in \mathbb{Z}_{\alpha}\right\}$ so that each set $\left\{x_{j}^{(i)}: j \in \mathbb{Z}_{6}\right\}$ induces a copy of $K_{6}^{*}$, and $\left(x_{j}^{(i)}, x_{s}^{(t)}\right)$ is an arc if and only if $i-t \equiv \pm 1(\bmod \alpha)$. We call an arc of the form $\left(x_{j}^{(i)}, x_{j+d}^{(i+1)}\right)$ a forward arc of difference $d$ from $x_{j}^{(i)}$, and $\left(x_{j}^{(i)}, x_{j+d}^{(i-1)}\right)$ a backward arc of difference $d$ from $x_{j}^{(i)}$. Again, by the mirror image of a resolution class we mean the image under the permutation $\left(x_{0} y_{0}\right)\left(x_{1} y_{1}\right) \ldots\left(x_{5} y_{5}\right)$.

We begin by defining the following directed 6-cycles, for all $i \in \mathbb{Z}_{\alpha}$ :

$$
\begin{aligned}
C_{00}^{(i)} & =x_{0}^{(i)} x_{0}^{(i+1)} x_{1}^{(i)} x_{1}^{(i+1)} x_{2}^{(i)} x_{2}^{(i+1)} x_{0}^{(i)}, \\
C_{11}^{(i)} & =x_{3}^{(i)} x_{3}^{(i+1)} x_{4}^{(i)} x_{4}^{(i+1)} x_{5}^{(i)} x_{5}^{(i+1)} x_{3}^{(i)}, \\
C_{01}^{(i)} & =x_{0}^{(i)} x_{3}^{(i+1)} x_{1}^{(i)} x_{4}^{(i+1)} x_{2}^{(i)} x_{5}^{(i+1)} x_{0}^{(i)} .
\end{aligned}
$$

Obtain the mirror image $D_{s}^{(i)}$ from $C_{s}^{(i)}$ by interchanging superscripts $i$ and $i+1$ for all vertices (see Figure 3).

We now form the following resolution classes.

$$
\begin{aligned}
& R_{1}=\left\{C_{00}^{(0)}, C_{11}^{(1)}, C_{00}^{(2)}, \ldots, C_{11}^{(\alpha-2)}, C_{01}^{(\alpha-1)}\right\}, \\
& R_{2}=\left\{C_{01}^{(0)}, C_{00}^{(1)}, C_{11}^{(2)}, \ldots, C_{00}^{(\alpha-2)}, C_{11}^{(\alpha-1)}\right\}, \\
& R_{3}=\left\{C_{11}^{(0)}, C_{01}^{(1)}, C_{01}^{(2)}, \ldots, C_{01}^{(\alpha-2)}, C_{00}^{(\alpha-1)}\right\} .
\end{aligned}
$$

We obtain three more resolution classes $R_{4}, R_{5}, R_{6}$ from the above by taking the mirror image of each directed 6-cycle. In Figure 3, the number next to a vertex on the left (right) indicates the forward (backward) difference of the arc from that vertex used by the given directed cycle. Observe that for all $i \in \mathbb{Z}_{\alpha}$, the following forward arcs have been used:

$$
\begin{array}{ll}
\left(x_{j}^{(i)}, x_{j+d}^{(i+1)}\right) & \text { for } \quad j=0,1 \text { and } d=0,1,3 ; \\
\left(x_{2}^{(i)}, x_{2+d}^{(i+1)}\right) & \text { for } \quad d=0,3,4 ; \\
\left(x_{j}^{(i)}, x_{j+d}^{(i+1)}\right) & \text { for } \quad j=3,4,5 \text { and } d=0,1,4 .
\end{array}
$$



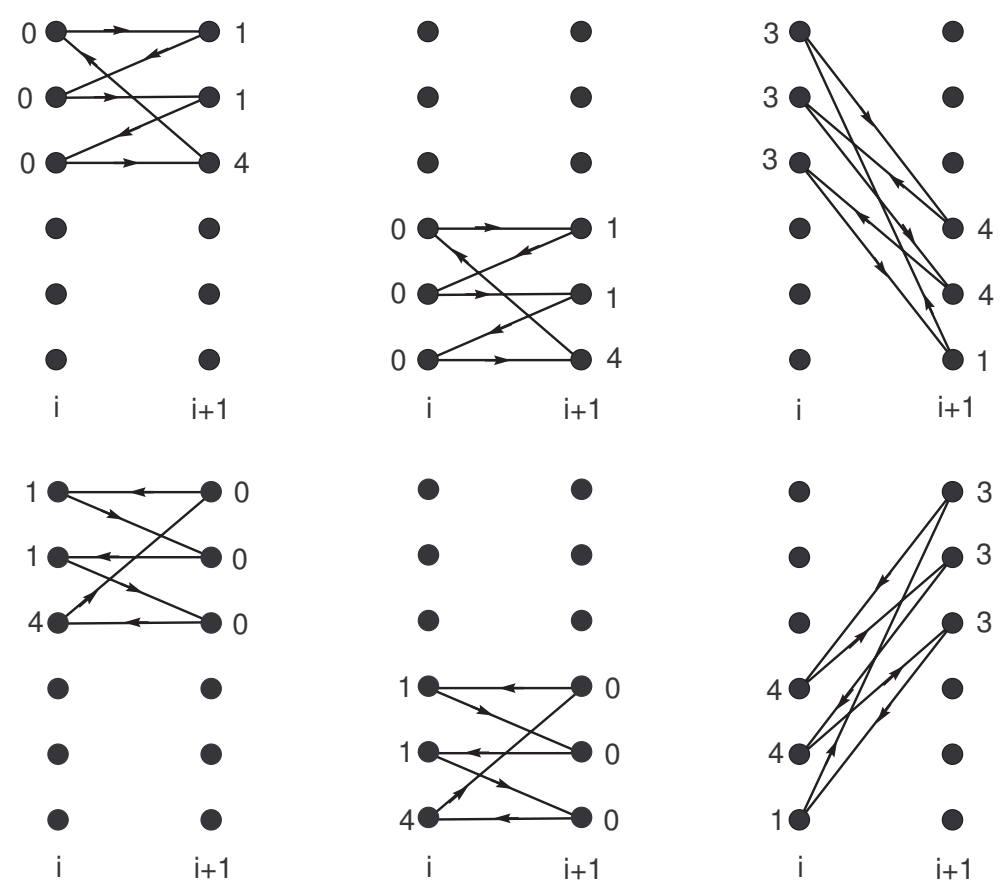

Figure 3: Directed 6-cycles $C_{00}^{(i)}, C_{11}^{(i)}$, and $C_{01}^{(i)}$ (top row), and their mirror images $D_{00}^{(i)}, D_{11}^{(i)}$, and $D_{01}^{(i)}$ (bottom row), with corresponding forward (left) and backward (right) arc differences.

Because of symmetry, backward arcs of the same differences have been used as well. This leaves us with forward and backward arcs of differences $2,4,5$ from $x_{j}^{(i)}$ for $j=0,1$; differences $1,2,5$ from $x_{2}^{(i)}$; and differences $2,3,5$ from $x_{j}^{(i)}$ for $j=3,4,5$.

To describe the next seven resolution classes, we first define the following directed 6-cycles (for all $i \in \mathbb{Z}_{\alpha}$ ) - see Figure 4:

$$
\begin{aligned}
C_{1}^{(i)} & =x_{0}^{(i)} x_{2}^{(i+1)} x_{4}^{(i)} x_{3}^{(i+1)} x_{5}^{(i)} x_{1}^{(i+1)} x_{0}^{(i)}, \\
C_{2}^{(i)} & =x_{0}^{(i)} x_{4}^{(i+1)} x_{1}^{(i)} x_{5}^{(i+1)} x_{2}^{(i)} x_{3}^{(i+1)} x_{0}^{(i)}, \\
C_{3}^{(i)} & =x_{1}^{(i)} x_{0}^{(i+1)} x_{2}^{(i)} x_{4}^{(i+1)} x_{3}^{(i)} x_{5}^{(i+1)} x_{1}^{(i)}, \\
C_{4}^{(i)} & =x_{3}^{(i)} x_{0}^{(i+1)} x_{4}^{(i)} x_{1}^{(i+1)} x_{5}^{(i)} x_{2}^{(i+1)} x_{3}^{(i)}, \\
C_{5}^{(i)} & =x_{0}^{(i)} x_{5}^{(i+1)} x_{4}^{(i)} x_{1}^{(i)} x_{3}^{(i+1)} x_{2}^{(i)} x_{0}^{(i)}, \\
C_{6}^{(i)} & =x_{1}^{(i)} x_{4}^{(i)} x_{0}^{(i+1)} x_{5}^{(i)} x_{3}^{(i)} x_{2}^{(i+1)} x_{1}^{(i)}, \\
C_{7}^{(i)} & =x_{0}^{(i)} x_{2}^{(i)} x_{1}^{(i+1)} x_{3}^{(i)} x_{5}^{(i)} x_{4}^{(i+1)} x_{0}^{(i)} .
\end{aligned}
$$

Observe that these directed 6-cycles jointly use up all leftover forward and backward arcs (as well as a bidirected 1-factor from each copy of $K_{6}^{*}$ ). Since for all $i \in \mathbb{Z}_{\alpha}$ and all 

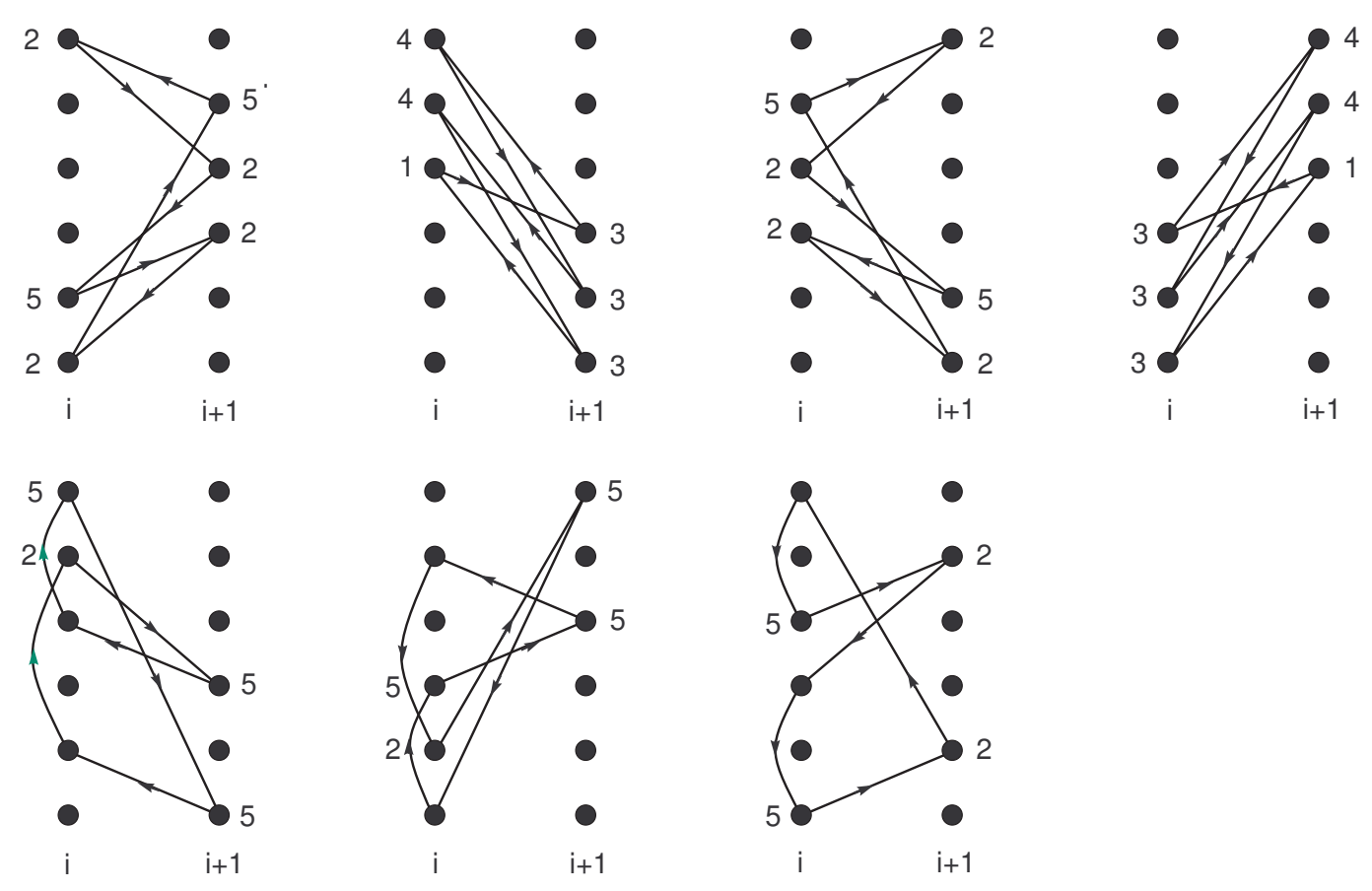

Figure 4: Directed 6-cycles $C_{j}^{(i)}$ for $j=1,2, \ldots, 7$, with corresponding forward (left) and backward (right) arc differences.

$j=1,2, \ldots, 7$, the directed 6 -cycles $C_{j}^{(i)}$ and $C_{j}^{(i+1)}$ are vertex-disjoint, we may form resolution classes $R_{j}^{\prime}$ as

$$
R_{j}^{\prime}=\left\{C_{j}^{(i)}: i=0,1, \ldots, \alpha-1\right\}
$$

What remains is a digraph consisting of $\alpha$ vertex-disjoint copies of $\left(K_{6}-I\right)^{*}$, which possesses an $\mathrm{R} \vec{C}_{6}$-D as a consequence of Theorem 11 and Lemma 9.

\section{Odd cycle length}

Our partial result in the case of odd cycle length (Theorem 20 below) heavily relies on Theorems 10 [4] for $\alpha$ odd, and 13 [13] for $\alpha$ even.

Theorem 20. Let $m \geqslant 5$ be an odd integer, and $\alpha$ a positive integer. Then:

1. If $\alpha$ is odd, then $K_{\alpha m}^{*}$ admits an $R \vec{C}_{m}-D$.

2. If $\alpha \equiv 0(\bmod 4)$, except possibly for $\alpha=8$, then $K_{\alpha m}^{*}$ admits an $R \vec{C}_{m}-D$.

3. If there exists an $R \vec{C}_{m}-D$ of $K_{2 m}^{*}$, then there exists an $R \vec{C}_{m}-D$ of $K_{\alpha m}^{*}$ for all even $\alpha$. 
Proof. 1. Assume $\alpha$ is odd. By Theorem 10, there exists an $\mathrm{R} C_{m}$-D of $K_{\alpha m}$. Directing each cycle in this decomposition once in each possible direction yields an $\mathrm{R} \vec{C}_{m}$-D of $K_{\alpha m}^{*}$

2. For $\alpha=4$, decompose

$$
K_{4 m}^{*}=\left(\bar{K}_{4} \succ K_{m}^{*}\right) \oplus\left(K_{4}^{*} \prec \bar{K}_{m}\right) .
$$

By a result of [10], the digraph $K_{4}^{*} \prec \bar{K}_{m}$ admits an $\mathrm{R} \vec{C}_{m}$-D, and by Theorem 12 , so does $\bar{K}_{4} \succ K_{m}^{*}$. Hence $K_{4 m}^{*}$ admits an $\mathrm{R} \vec{C}_{m}$-D.

For $\alpha=4 \beta$ with $\beta \geqslant 3$, first decompose

$$
K_{4 \beta m}^{*}=\left(\bar{K}_{\beta} \succ K_{4 m}^{*}\right) \oplus\left(K_{\beta}^{*} \succ \bar{K}_{4 m}\right) .
$$

Since $K_{\beta}^{*} \succ \bar{K}_{4 m}$ admits an $\mathrm{R} \vec{C}_{m}$-D as a corollary of Theorem 13 and Lemma 9, and $\bar{K}_{\beta} \succ K_{4 m}^{*}$ admits an $\mathrm{R} \vec{C}_{m}$-D by the previous paragraph, we have the result.

3. Finally, let $\alpha=2 \beta$ with $\beta \geqslant 3$. Decompose

$$
K_{2 \beta m}^{*}=\left(\bar{K}_{\beta} \succ K_{2 m}^{*}\right) \oplus\left(K_{\beta}^{*} \succ \bar{K}_{2 m}\right) .
$$

By Theorem 13, there exists an $\mathrm{R} C_{m}$-D of $K_{\beta} \imath \bar{K}_{2 m}$, and hence Lemma 9 guarantees the existence of an $\mathrm{R} \vec{C}_{m}$-D of $K_{\beta}^{*} \chi \bar{K}_{2 m}$. Therefore, if there exists an $\mathrm{R} \vec{C}_{m}$-D of $K_{2 m}^{*}$, there also exists an $\vec{R}_{m^{-}}$D of $K_{\alpha m}^{*}$ for any even $\alpha \geqslant 6$.

In the case of odd cycle length $m \geqslant 5$, it thus remains to consider the case $\alpha=2$. In part assisted by a computer search, we have so far been able to verify the following.

Lemma 21. [8] An $R \vec{C}_{m}-D$ of $K_{2 m}^{*}$ exists for:

- all odd $m$ such that $5 \leqslant m \leqslant 49$ and $m \neq \equiv 0(\bmod 3)$, and

- all odd $m$ such that $5 \leqslant m \leqslant 27$ and $m \equiv 0(\bmod 3)$.

We therefore propose the following conjecture. Recall that an $\mathrm{R} \vec{C}_{3}$-D of $K_{6}^{*}$ does not exist by Theorem 1 [7].

Conjecture 22. Let $m$ be a positive odd integer. Then $K_{2 m}^{*}$ admits an $\vec{R}_{m}$-D if and only if $m \geqslant 5$. 


\section{Some related results}

In this section we present two resolvable directed cycle decompositions of $K_{n}^{*}$ that are "close" to being $\mathrm{R} \vec{C}_{m}$-D. The following term will be convenient to use: a directed 2-factor of a digraph $D$ is a spanning subdigraph of $D$ that is a vertex-disjoint union of directed cycles. We shall write $\vec{F}(\alpha * m)$ to denote a directed 2-factor consisting of $\alpha$ pairwise vertex-disjoint directed $m$-cycles. Thus an $\mathrm{R} \vec{C}_{m}$-D is a decomposition into directed 2factors, each isomorphic to $\vec{F}(\alpha * m)$, for an appropriate integer $\alpha$. The directed 2factorizations of $K_{n}^{*}$ constructed below will be such that only one directed 2-factor is not an $\vec{F}(\alpha * m)$. The first of these factorizations is a quick corollary of Theorem 11 .

Proposition 23. Let $m \geqslant 2$ and $\alpha \geqslant 1$ be integers. Then $K_{2 \alpha m}^{*}$ can be decomposed into directed 2-factors of which one is an $\vec{F}(\alpha m * 2)$ and the rest are $\vec{F}(2 \alpha * m)$.

Proof. The result is obvious for $m=2$. For $m \geqslant 3$, there exists an $\mathrm{R}_{m}$ - $\mathrm{D}$ of $K_{2 \alpha m}-I$ by Theorem 11. Direct each $m$-cycle in this decomposition in each of the two possible ways to obtain an $\mathrm{R} \vec{C}_{m}$-D of $\left(K_{2 \alpha m}-I\right)^{*}$. Finally, replace each edge in the missing 1 -factor $I$ in $\left(K_{2 \alpha m}-I\right)^{*}$ by a directed 2-cycle to obtain a directed 2-factor $\vec{F}(\alpha m * 2)$.

The following result is as close to Conjecture 22 for every odd $m$ as we can get at present.

Theorem 24. Let $m \geqslant 3$ be an odd integer.

1. If $m \equiv 0(\bmod 3)$, then $K_{2 m}^{*}$ can be decomposed into directed 2-factors of which one is an $\vec{F}\left(3 * \frac{2 m}{3}\right)$ and the rest are $\vec{F}(2 * m)$.

2. Otherwise, $K_{2 m}^{*}$ can be decomposed into directed 2-factors of which one is an $\vec{F}(1 *$ $2 m$ ) and the rest are $\vec{F}(2 * m)$.

Proof. Let $m=2 k+1$, and let $\{X, Y\}$ be a partition of the vertex set of $K_{2 m}^{*}$ with $X=\left\{x_{0}, x_{1}, \ldots, x_{2 k}\right\}$ and $Y=\left\{y_{0}, y_{1}, \ldots, y_{2 k}\right\}$. Arcs of the form $\left(x_{i}, x_{i}+d\right)$ and $\left(y_{i}, y_{i}+d\right)$, for $d=1,2, \ldots, 2 k$, will be called of left and right, respectively, pure difference $d$. Arcs of the form $\left(x_{i}, y_{i+d}\right)$ and $\left(y_{i}, x_{i+d}\right)$, for $d=0,1, \ldots, 2 k$, will be called of left and right, respectively, mixed difference $d$. All subscripts are calculated modulo $2 k+1$.

Define directed $m$-cycles $C_{0}$ and $C_{0}^{\prime}$ as follows:

$$
C_{0}=x_{0} y_{0} x_{1} y_{1} x_{2} \ldots y_{k-1} x_{k} x_{0} \quad \text { and } \quad C_{0}^{\prime}=y_{k} x_{k+1} y_{k+1} x_{k+2} \ldots y_{2 k} y_{k} .
$$

Observe that the only arcs of left mixed difference 0 or right mixed difference $2 k$ not used in these two cycles are $\left(x_{k}, y_{k}\right)$ and $\left(y_{2 k}, x_{0}\right)$. For $i=1,2, \ldots, 2 k$, obtain directed $m$-cycles $C_{i}$ and $C_{i}^{\prime}$ from $C_{0}$ and $C_{0}^{\prime}$, respectively, by adding $i$ to the subscript of each vertex in $X$ and adding $2 i$ to the subscript of each vertex in $Y$. Thus directed $m$-cycles $C_{i}$ and $C_{i}^{\prime}$ together use up all arcs of left mixed difference $i$ and right mixed difference $2 k+i$ except for $\left(x_{k+i}, y_{k+2 i}\right)$ and $\left(y_{2 k+2 i}, x_{i}\right)$. For $i=0,1,2, \ldots, 2 k$, let $F^{(i)}$ be the directed 2-factor in $K_{2 m}^{*}$ defined by $F^{(i)}=\left\{C_{i}, C_{i}^{\prime}\right\}$. 
Let $\left\{C_{X}^{1}, C_{X}^{2}, \ldots, C_{X}^{2 k}\right\}$ be an $\mathrm{R} \vec{C}_{m}$-D of the copy of $K_{m}^{*}$ induced by $X$, and similarly, let $\left\{C_{Y}^{1}, C_{Y}^{2}, \ldots, C_{Y}^{2 k}\right\}$ be an $\mathrm{R} \overrightarrow{C_{m}}$-D of the copy of $K_{m}^{*}$ induced by $Y$. Relabelling the vertices if necessary, we may assume that $C_{X}^{2 k}=x_{0} x_{-k} x_{-2 k} \ldots x_{k} x_{0}$ and $C_{Y}^{2 k}=y_{0} y_{-k} y_{-2 k} \ldots y_{k} y_{0}$. For $i=1,2, \ldots, 2 k-1$, let $F^{(2 k+i)}$ be the directed 2 -factor in $K_{2 m}^{*}$ defined by $F^{(2 k+i)}=$ $\left\{C_{X}^{i}, C_{Y}^{i}\right\}$.

Observe that arcs of pure difference $k+1$ (corresponding to directed $m$-cycles $C_{X}^{2 k}$ and $\left.C_{Y}^{2 k}\right)$ were used up in directed 2-factors $F^{(0)}, F^{(1)}, \ldots, F^{(2 k)}$. The only remaining arcs are hence those of the form $\left(x_{k+i}, y_{k+2 i}\right)$ and $\left(y_{2 k+2 i}, x_{i}\right)$, for $i=0,1,2, \ldots, 2 k$. It is clear that they form a directed 2 -factor - call it $F$. We'll show that it must be either of the form $\vec{F}(1 * 2 m)$ or $\vec{F}\left(3 * \frac{2 m}{3}\right)$.

One of the directed cycles in $F$ is

$$
C_{F}=x_{0} y_{k+1} x_{\frac{k}{2}+1} y_{2} x_{k+2} y_{k+4} \ldots x_{i\left(\frac{k}{2}+1\right)} y_{(i+1)(k+2)} \ldots x_{0}
$$

if $k$ is even, and

$$
C_{F}=x_{0} y_{k+1} x_{\frac{3}{2}(k+1)} y_{2} x_{k+2} y_{k+4} \ldots x_{i \frac{3}{2}(k+1)} y_{(i+1)(k+2)} \ldots x_{0}
$$

if $k$ is odd. Note that for even $k, \operatorname{gcd}\left(\frac{k}{2}+1,2 k+1\right)=\operatorname{gcd}(k+2,2 k+1)$ and for odd $k, \operatorname{gcd}\left(\frac{3}{2}(k+1), 2 k+1\right)=\operatorname{gcd}(k+2,2 k+1)$. Since $\operatorname{gcd}(k+2,2 k+1)$ is 3 if $2 k+1 \equiv 0$ $(\bmod 3)$ and 1 otherwise, the directed cycle $C_{F}$ is of length $\frac{2 m}{3}$ if $m \equiv 0(\bmod 3)$, and of length $2 m$ otherwise. It follows that the directed 2 -factor $F$ is of the form $\vec{F}\left(3 * \frac{2 m}{3}\right)$ in the first case, and $\vec{F}(1 * 2 m)$ in the second.

Corollary 25. Let $m \geqslant 3$ be an odd integer and $\alpha \neq 2$ a positive integer. Then $K_{2 \alpha m}^{*}$ can be decomposed into directed 2-factors of which one is an $\vec{F}\left(3 \alpha * \frac{2 m}{3}\right)$ if $m \equiv 0(\bmod 3)$, and $\vec{F}(\alpha * 2 m)$ otherwise, and the rest are $\vec{F}(2 \alpha * m)$.

Proof. For $\alpha=1$, the statement is identical to Theorem 24. Hence assume $\alpha \geqslant 3$. Now decompose

$$
K_{2 \alpha m}^{*}=\bar{K}_{\alpha} \succ K_{2 m}^{*} \oplus K_{\alpha}^{*} \succ \bar{K}_{2 m} .
$$

It follows from Theorem 13 and Lemma 9 that there exists a $\mathrm{R} \vec{C}_{m}$-D of $K_{\alpha}^{*} \imath \bar{K}_{2 m}$. On the other hand, Theorem 24 shows that $\bar{K}_{\alpha} \prec K_{2 m}^{*}$ can be decomposed into directed 2-factors of which one is an $\vec{F}\left(3 \alpha * \frac{2 m}{3}\right)$ if $m \equiv 0(\bmod 3)$, and $\vec{F}(\alpha * 2 m)$ otherwise, and the rest are $\vec{F}(2 \alpha * m)$. Putting these two decompositions together results in a desired directed 2-factorization.

\section{Acknowledgements}

The authors gratefully acknowledge support by the Natural Sciences and Engineering Research Council of Canada. 


\section{References}

[1] P. Adams and D. Bryant. Resolvable directed cycle systems of all indices for cycle length 3 and 4. Unpublished.

[2] B. Alspach, H. Gavlas, M. Šajna, and H. Verrall. Cycle decompositions IV: Complete directed graphs and fixed length directed cycles. J. Combin. Theory Ser. A, 103:165208, 2003.

[3] B. Alspach and R. Häggkvist. Some observations on the Oberwolfach problem. J. Graph Theory, 9:177-187, 1985.

[4] B. Alspach, P. J. Schellenberg, D. R. Stinson, and D. Wagner. The Oberwolfach problem and factors of uniform odd length cycles. J. Combin. Theory Ser. A, 52:20 43, 1989.

[5] F. E. Bennett and X. Zhang. Resolvable Mendelsohn designs with block size 4. Aequationes Math., 40:248-260, 1990.

[6] J.-C. Bermond, O. Favaron, and M. Mahéo. Hamiltonian decomposition of Cayley graphs of degree 4. J. Combin. Theory Ser. B, 46:142-153, 1989.

[7] J.-C. Bermond, A. Germa, and D. Sotteau. Resolvable decomposition of $K_{n}^{*}$. J. Combin. Theory Ser. A, 26:179-185, 1979.

[8] A. Burgess, N. Francetić, P. Niesink, and M. Šajna. On the directed Oberwolfach Problem with equal cycle lengths: the odd case. In preparation.

[9] C. J. Colbourn and J. H. Dinitz (editors). Handbook of combinatorial designs. Chapman and Hall/CRC, Boca Raton, FL, 2007.

[10] N. Francetić and M. Šajna. On the directed Oberwolfach Problem for complete equipartite digraphs. In preparation.

[11] D. G. Hoffman and P. J. Schellenberg. The existence of $C_{k}$-factorizations of $K_{2 n}-F$. Discrete Math., 97:243-250, 1991.

[12] C. Huang, A. Kotzig, and A. Rosa. On a variation of the Oberwolfach problem. Discrete Math., 27:261-277, 1979.

[13] J. Liu. The equipartite Oberwolfach problem with uniform tables. J. Combin. Theory Ser. A, 101:20-34, 2003.

[14] E. Lucas. Récréations Mathématiques, Vol. II. Gauthier-Villars, Paris, 1892.

[15] T.W. Tillson. A hamiltonian decomposition of $K_{2 m}^{*}, 2 m \geqslant 8$. J. Comb. Theory Ser. $B, 29: 69-74,1980$. 